\title{
Ce que les expériences extraprofessionnelles font à la pratique professionnelle
}

\author{
Le cas de l'écrivaine Amélie Nothomb
}

\begin{abstract}
Émilie Saunier ${ }^{1}$
[Résumé] À partir de l'analyse de la pratique littéraire d'Amélie Nothomb et de ses déterminants sociaux, notre article vise à montrer la portée heuristique d'une ouverture du champ d'observation de cette activité - que l'on peut qualifier de « professionnelle » - à des instances de socialisation extra-professionnelles. La trajectoire littéraire d'Amélie Nothomb, sa manière d'investir sa pratique d'écriture et de la publiciser ne peuvent se comprendre si l'on fait fi d'un certain nombre d'expériences extralittéraires vécues en amont de son entrée dans le champ littéraire et qui ont produit des effets sur son activité littéraire. Dans cette perspective, sa création littéraire ne peut se réduire aux seuls enjeux du champ littéraire dans la mesure où la romancière fait travailler dans son activité d'écriture un certain nombre de préoccupations et de questions qui la caractérisent en tant qu'individu socialement "fabriqué ». Nous montrerons notamment comment l'étude des expériences extraprofessionnelles de l'écrivaine, tout à la fois socialement situées et relativement singulières, permet d'éclairer le contenu thématique et stylistique de sa production littéraire, en prenant l'exemple du rapport particulier au langage mis en scène dans ses récits.
\end{abstract}

Mots-clés : création littéraire ; expériences socialisatrices ; schèmes littéraires ; transposition; Amélie Nothomb.

[Abstract] This paper is based on the study of Amélie Nothomb's literary practice and its social motors. Its purpose is to show the heuristic scope of broadening the focus of observation of such a "professional" activity to extra-professional agents of socialization. Understanding Amélie Nothomb's literary path and her personal way of investing her literary and paraliterary activities requires studying the social configurations she went through - even before becoming a writer -, configurations that created and sustained her desire to write, but also shaped her way of seeing the world as well as the questions, and obsessions that characterized her as a socially "made" individual and which came to be expressed in a particular way in her novels. More precisely, I will show how the study of Amélie Nothomb's extra-professional experiences sheds light on thematic and stylistic aspects of her literary work, such as the particular relationship to language present in her writings.

Keywords: literary creation; socializing experiences; literary schemes; transposition; Amélie Nothomb.

1 Éditions, langages, littératures, informatique, arts, didactiques, discours (EA 4661), Université de Franche-Comté. 


\section{Introduction}

Notre contribution s'appuie sur un travail de thèse en sociologie qui vise à éclairer la pratique littéraire d'Amélie Nothomb (Saunier, 2012). De façon générale, l'univers littéraire ne peut être trop rapidement défini comme un univers « professionnel ». En effet, il ne peut exercer une force socialisatrice forte et identique sur tous les individus qui le fréquentent dans la mesure où ceux qui s'y investissent le font à des degrés divers (Lahire, 2006). En outre, si cet univers est structuré par des traditions, des propriétés et des règles spécifiques, son statut et ses règles sont peu institutionnalisées relativement à d'autres domaines professionnels comme la profession médicale par exemple, car " aucune formation, aucun diplôme ni aucun concours ne viennent baliser de façon nette les parcours [littéraires] au point de pouvoir donner à l'ensemble de ceux qui y participent des signes certains d'appartenance » (Lahire, 2006 : 40). Il apparaît donc difficile de parler de manière générale de " $\operatorname{profession}^{2}$ » ou de « métier » auquel se rattacheraient des conditions d'entrée clairement identifiables et un statut économique stable pour qualifier l'activité littéraire des écrivains (Menger, 2009).

Néanmoins, en ce qui concerne Amélie Nothomb, on peut considérer son activité littéraire comme un espace de socialisation professionnelle. En effet, elle a pu, dès ses débuts en 1992, vivre de ses publications littéraires et en obtenir des revenus réguliers. Cette activité est subjectivement investie par la romancière à la fois comme une vocation et comme une profession à part entière. Elle accorde de longues heures quotidiennes à faire la promotion de ses romans, à appeler ses lecteurs et à répondre à leurs lettres, ainsi qu'à participer à des salons et à des rencontres. À sa demande, la maison d'édition Albin Michel lui a donné un bureau qu'elle occupe très régulièrement et dans lequel elle répond aux courriers et aux interviews à l'occasion de la publication, chaque année, de son nouveau roman: "Disons que je suis toujours une petite employée de bureau japonaise, qui rend ses devoirs chaque année. Ce rythme "scolaire" me convient ${ }^{3}$ ». Elle dit « pleinement accepter les règles du jeu » de la critique ${ }^{4}$ et de la promotion annuelle de ses livres ${ }^{5}$. Amélie Nothomb peut être considérée comme une « joueuse professionnelle » : cet investissement est « le fait des écrivains "à succès" dont la production relève même parfois de la "littérature industrielle", et qui ont des ventes

\footnotetext{
${ }^{2}$ Au sens du Beruf, que définit Max Weber : « Nous appellerons "profession" [Beruf] la spécification, la spécialisation et la combinaison de prestations qui permettent à une personne de s'assurer des chances permanentes d'approvisionnement et de gains. » (Weber, 1995 : 201).

${ }^{3}$ Livres Hebdo, 2 juillet 2004.

${ }^{4}$ Émission « Sous la couette de Jessica », sur la radio Le Mouv', 18 novembre 2006.

${ }^{5}$ « Mon roman est en vente depuis le $1^{\text {er }}$ septembre. En vente : c'est à dessein que j'ai choisi une expression aussi crue. II ne faut pas se leurrer sur la nature de l'édition. Mais j'ai décidé de jouer sans états d'âme un jeu qui ne méritait aucune considération profonde. Tant d'écrivains essaient d'être publiés, en vain : j'y suis parvenue [...]. Au nom de quoi oserais-je jouer la carte de la profondeur blessée ? » (Le Carnet et les Instants, $n^{\circ} 75,15$ novembre 1992).
} 
de livres leur permettant de vivre des droits d'auteur »(Lahire, 2006: 167). Grâce aux succès commerciaux de ses premiers romans, l'univers littéraire devient rapidement pour Amélie Nothomb un espace de socialisation professionnelle dans la mesure où elle entretient des liens réguliers et fréquents avec le milieu littéraire (et médiatique). Néanmoins, d'autres socialisations préexistent à cette socialisation professionnelle : il revient à la sociologie de la littérature la tâche de penser l'articulation de ces deux temps de la socialisation « primaire » et « secondaire » (Berger et Luckmann, 1996). Plus précisément, la prise en compte des expériences antérieures et extralittéraires d'Amélie Nothomb permet d'observer que celle-ci (re)joue sur la scène littéraire des préoccupations, obsessions ou expériences vécues en amont de son entrée dans le champ littéraire : ses textes donnent à voir, à travers des codes de transposition propres au travail littéraire, un point de vue singulier sur le monde social. Il s'agit alors, à travers le cas d'Amélie Nothomb, de rendre compte de "ce dont [l'écrivain] est luimême traversé, ou préoccupé » (Viart, 2007: 11) et de la «forme» que prend cette manière de parler du monde ou de soi. Nous montrerons que certaines expériences socialisatrices extraprofessionnelles d'Amélie Nothomb permettent d'éclairer ce qui est travaillé dans son écriture littéraire en prenant l'exemple du rapport particulier au langage mis en scène dans ses récits.

Encadré méthodologique

L'enjeu de notre travail est de tisser des liens entre des propriétés de la vie d'Amélie Nothomb et celles de ses textes qui soient pertinents, c'est-à-dire non assimilables à un « reflet $^{6}{ } »$ ou à des liens trop rapides entre des anecdotes sur la vie de l'écrivaine et ses textes. Cela nécessite de reconstruire les éléments structurant d'une part la vie d'Amélie Nothomb et d'autre part ses textes.

Concernant ces derniers, nous nous sommes concentrée sur les seize textes publiés aux éditions Albin Michel de 1992 à 2007. Nous avons reconstitué des séries à partir d'une recherche systématique de récurrences dans les éléments structurant les textes. Une lecture des commentaires réalisés par les critiques littéraires ainsi que les chercheurs en littérature sur ces textes a, dans un premier temps, permis de repérer des propriétés stylistiques et thématiques. Afin de décrire et d'analyser les narrations, nous nous sommes inspirée des principes de l'analyse structurale et typologique du récit telle qu'elle a pu être mise en œuvre par Barthes sur les récits littéraires (Barthes, 1966) ou encore Propp sur le conte (Propp, 1965). L'approche structurale des œuvres n'était pas le but ultime de la démarche car notre compréhension des œuvres visait à articuler la lecture des textes avec les contextes sociaux

${ }^{6}$ Bourdieu a notamment montré les limites d'une approche marxiste des œuvres dans la mesure où elle les pense "comme simple reflet ou comme "expression symbolique" du monde social », en les « rapport[ant]directement aux caractéristiques sociales des auteurs ou des groupes qui en étaient les destinataires déclarés ou supposés, qu'elles sont censées exprimer » (Bourdieu, $1992: 284$ ). 
de leur création. Sur ce point, la manière dont Panofsky a cherché à reconstruire « l'habitude mentale » ou le « principe » qui « règle l'acte » du créateur à travers une étude de ses œuvres (Panofsky, 1967 : 83) nous a aidée à reconstruire les schèmes spécifiques de perception, de catégorisation et d'interprétation du monde social mis en œuvre par Amélie Nothomb dans sa pratique d'écriture littéraire.

En outre, l'identification des expériences sociales qui ont marqué l'écrivaine et des traces qu'elles ont laissé sous la forme de dispositions ou de compétences a nécessité de recourir à des dispositifs variés. Tout d'abord, la passation d'entretiens semi-directifs avec Amélie Nothomb : nous avons pu la rencontrer à deux reprises dans son «bureau » des éditions Albin Michel à Paris, pour une durée totale de cinq heures. Par ailleurs, parce qu'Amélie Nothomb est une écrivaine d'origine belge qui a construit sa carrière en France, nous avons dépouillé ses interviews données dans ces deux pays afin de recueillir des informations sur ses propriétés sociales et sa trajectoire sociale et littéraire, ainsi que de décrire la manière dont elle se présente publiquement en tant qu'écrivaine. À ces éléments s'ajoute une étude de la réception de ses œuvres par des critiques journalistiques et académiques : la finalité était d'étudier les profits de l'écrivaine du point de vue de leurs effets sur la pratique d'écriture d'Amélie Nothomb et sur sa manière de se définir. En outre, afin de "contrôler» les données factuelles recueillies auprès d'Amélie Nothomb, de mettre en rapport ses paroles avec d'autres aspects de la réalité non évoqués par elle, et de recueillir des informations sur ses manières d'être et de se comporter dans divers domaines de son existence (en prenant en compte de possibles évolutions au cours du temps), nous avons réalisé des entretiens et engagé des échanges écrits avec des individus s'inscrivant dans divers cercles de son existence passée ou présente (membres de sa famille, membres de son cercle amical et professionnel, anciens élèves et enseignants). Enfin, la consultation d'archives a permis le recueil d'informations supplémentaires et de première main afin d'établir une chronologie d'événements factuels (événements, dates, lieux).

\section{Retour sur notre projet de connaissance}

Le présent article s'inscrit dans un projet plus général visant à montrer que pour comprendre finement ce qui se joue au sein d'une activité professionnelle (et ici plus spécifiquement artistique), on ne peut faire fi d'une analyse approfondie des instances de socialisation extra professionnelles (artistiques). Dans son travail sur Mozart, Elias soulignait que l'individu n'est pas composé « de tiroirs séparés dont l'un serait celui de l'artiste, l'autre celui de l'individu humain» (Elias, 1991 : 136) : des expériences extraartistiques peuvent pénétrer le domaine artistique et se trouver traduites selon les règles propres à cet espace. Notre perspective vise alors à resituer l'activité professionnelle littéraire d'Amélie Nothomb dans l'histoire de ses socialisations successives et dans sa trajectoire sociale. Pourquoi est-elle attirée vers cette activité à un moment donné de sa vie ? Qu’y investit-elle ? Comment la définit-elle ? Qu'Amélie Nothomb fait-elle "travailler» de ses expériences vécues dans l'écriture littéraire? 
Qu'est-ce qui est en jeu dans l'activité littéraire et qui lui donne ses formes et ses fonctions spécifiques?

Bourdieu propose une approche sociologique de ces questions en élaborant un raisonnement qui peut être synthétisé sous la forme d'un dispositif tripode entre les dispositions, les positions et les prises de position (Bourdieu, 1992). Cela consiste à observer comment les dispositions d'un écrivain génèrent à l'intérieur du champ littéraire une position particulière et comment cette position peut rendre compte de ses prises de position dans le champ. Bourdieu se propose par ce biais de dépasser les approches externes et internes de la littérature tout en évitant l'écueil du reflet : les propriétés sociales des écrivains n'expliquent pas « directement » leur création mais sont médiatisées par le champ littéraire. Cette approche a fait l'objet de prolongements qui reposent toutes sur l'hypothèse d'une homologie entre l'espace des positions et l'espace des prises de position et qui font du champ littéraire un principe explicatif central de la création littéraire (Boschetti, 2001 ou encore, dans le cadre d'analyses sociopoétiques, Meizoz, 2007).

Une telle démarche présente plusieurs limites. Postuler une homologie structurale entre l'œuvre et la position de son auteur dans le champ littéraire présente tout d'abord le risque de "réduire l'œuvre au statut de support expressif de la position d'un auteur » (Fabiani, 1999 : 86). En effet, penser que c'est l'état du rapport de forces dans le champ et les liens objectifs qui relient l'écrivain aux autres agents du champ qui déterminent ses prises de position dans le champ signifie que ses œuvres pourraient se déduire, dans leurs thèmes et leur style, de ce que les autres œuvres ne sont pas. Or, ce que l'œuvre contient dans ses thèmes ne peut être seulement saisi comme un " coup » joué par l'écrivain dans le champ littéraire ou à une volonté de se distinguer de tel écrivain ou de tel courant. Des préoccupations d'ordre extralittéraires peuvent aussi être activées et transposées dans les contraintes propres à l'ordre littéraire (Lahire, 2010). Une autre limite que l'on peut identifier réside dans les écarts entre cette approche en termes de dispositif tripode de Bourdieu et l'analyse qu'il a produite de L'Éducation sentimentale (Bourdieu, 1992). En effet, dans son étude, il met l'accent sur la position occupée par l'écrivain dans le champ littéraire afin d'expliquer ses œuvres, aux dépens d'une analyse de son habitus conduite en amont. Celui-ci se trouve souvent réduit à des informations assez générales : quelques propriétés sociales, une position sociale, une pente de trajectoire.

Nous avons alors veillé, dans notre interprétation sociologique des prises de position littéraires d'Amélie Nothomb, à prendre en compte tout à la fois ses dispositions et compétences construites en amont ou en parallèle de sa socialisation professionnelle et ses dispositions et compétences acquises dans le champ littéraire. Cela a impliqué de hiérarchiser les diverses socialisations de l'écrivaine et de les appréhender dans leur chronologie. Dans le cadre de ce projet, nous avons déjà pu montrer dans des articles précédents comment les modalités d'entrée d'Amélie Nothomb dans le champ littéraire étaient intimement liées à ses propriétés et ressources accumulées lors d'expériences 
plurielles de socialisation en développant les dimensions de l'origine sociale, de la nationalité et du genre. Sa socialisation au sein d'une famille de la noblesse culturelle belge $^{7}$ et sa socialisation universitaire ont conditionné en partie la nature de ses aspirations professionnelles, sa connaissance du champ littéraire français et la manière dont elle s'y est positionnée (Saunier, 2015a). Nous avons également identifié des conditions dispositionnelles (aristocratiques, intellectuelles, culturelles et littéraires, ainsi qu'une aisance verbale et sociale) construites en amont du champ littéraire, rendant possible un jeu « sur les deux tableaux » et permettant de cumuler des profits tout à la fois symboliques et médiatico-économiques (Saunier, 2015b). Aussi ces différentes études ont-elles pu mettre en avant le rôle des expériences socialisatrices extra-artistiques sur la trajectoire professionnelle d'Amélie Nothomb ainsi que sa manière particulière d'investir sa pratique d'écriture et de la publiciser sur la scène littéraire (Giraud, Saunier, 2012).

Que dire désormais de la manière personnelle dont l'écrivaine s'exprime dans sa pratique même d'écriture, autrement dit dans les contenus mêmes des textes? Nous avons choisi ici de centrer notre propos sur une dimension particulière des textes, celle de la place accordée à la parole des personnages. L'analyse des textes d'Amélie Nothomb révèle une croyance de l'écrivaine dans le pouvoir performatif du langage, que celui-ci prenne des formes orales (la parole en situation) ou écrites (l'écriture ordinaire ou littéraire). Dans son analyse des propriétés du langage ordinaire, Austin envisage les énoncés "performatifs» comme ayant la capacité de "faire» ce qu’ils disent en le disant ; ils engagent le locuteur ou déterminent l'action d'autrui (Austin, 1991). Austin parle "d'actes de langage». Nous définissons la croyance en sa performativité comme la croyance en la capacité du langage à agir sur soi (se sauver, se guérir, se former) et à agir sur les autres (les séduire, les convaincre, les blesser). $\mathrm{Si}$ l'on voulait rapporter ce type d'observation à une question qui structure les textes d'Amélie Nothomb, on pourrait donc dire que ce qui est interrogé dans ses récits, c'est la capacité du langage à agir sur soi et sur les autres. Ce questionnement est très présent dans le monde littéraire: il se situe au cœur des réflexions en littérature (cf. par exemple Larthomas, 2001) et la croyance en un pouvoir des mots se rencontre particulièrement chez les écrivains, que ce soit dans leurs discours ou dans leurs textes.

\footnotetext{
${ }^{7}$ Du point de vue de leur ancienneté dans la noblesse et de leurs titres, les familles ScheyvenBoucher (les branches maternelles de l'écrivaine) et Nothomb-Lancksweert (ses branches paternelles) appartiennent au même type de noblesse modérément prestigieuse et plutôt orientée du côté du pôle culturel, surtout pour la famille Nothomb. L'influence des Nothomb s'est construite grâce à leur action politique et diplomatique depuis la naissance de la Belgique, laquelle action a fait l'objet de publications par plusieurs membres masculins de la famille. Des essais, mémoires et témoignages constituent une part importante des productions de cette famille, auxquelles s'ajoutent des romans historiques et de la poésie. Quant à la famille Scheyven, elle a également été présente sur la scène politique et diplomatique, et elle s'est distinguée par l'accumulation de capital économique au cours du XX' siècle.
} 
Cette croyance est particulièrement investie dans les œuvres de l'écrivaine et semble ainsi constituer la trace d'une socialisation (notamment professionnelle ${ }^{8}$ ) à cette idéologie littéraire. Reste que tous les écrivains ne mettent pas en scène cette croyance dans leurs textes, à travers des situations narratives et des formes stylistiques précises, comme c'est le cas chez Amélie Nothomb. Les manières spécifiques dans lesquelles cette question est travaillée sont lisibles en lien avec certaines expériences socialisatrices extra-littéraires. On voit donc que le modèle interprétatif par le champ littéraire et le modèle interprétatif par les expériences extralittéraires entrent tous deux en jeu, mais ce dernier permettrait d'examiner au plus près la spécificité avec laquelle ce topos est mis en scène littérairement. Nous avons choisi de ne traiter qu'une seule forme de langage, la parole. Après avoir montré, au travers de l'analyse sociologique de certains éléments biographiques, l'intériorisation par Amélie Nothomb d'une croyance en la performativité de la parole, nous montrerons comment ce schème de pensée se retraduit dans des codes propres à l'écriture littéraire.

\section{Des expériences extralittéraires propices à la genèse d'une croyance en la performativité de la parole}

La parole est ici appréhendée comme une activité déployée au cours d'échanges interindividuels. L'acte de parler et, plus précisément, de bien maîtriser et mesurer ses mots, a peu à peu été intériorisé par Amélie Nothomb comme une ressource importante, qui est susceptible de conférer du pouvoir à celui qui sait en faire usage.

\subsection{Une socialisation familiale marquée par une culture de l'excellence}

Ce point de vue particulier sur la parole se construit dans un cadre familial spécifique, marqué par une culture de l'excellence aristocratique qui fait de cette pratique un enjeu important dans les relations sociales. Amélie Nothomb est issue de deux familles de notables, les Nothomb et les Scheyven, connues en Belgique pour leur action politique et diplomatique ${ }^{9}$. Anoblies au $\mathrm{XX}^{\mathrm{e}}$ siècle, elles appartiennent à une noblesse modérément prestigieuse au regard des catégories de noblesse existantes en Belgique ${ }^{10}$. Le père d'Amélie Nothomb, Patrick Nothomb, a effectué une brillante carrière de

\footnotetext{
${ }^{8}$ Antérieurement à sa socialisation professionnelle, nous pouvons faire l'hypothèse que ce rapport particulier au langage a pu être intériorisé par Amélie Nothomb au cours de ses études de philologie romane, à l'Université libre de Bruxelles, de 1984 à 1988, c'est-à-dire peu avant son entrée dans le champ littéraire.

${ }_{9}^{9}$ Citons à titre d'exemple la participation de Jean-Baptiste Nothomb à la rédaction de la Constitution Belge. La famille Scheyven a aussi été présente sur la scène politique et diplomatique, mais de manière plus ponctuelle.

${ }^{10}$ Cf. la typologie des catégories de noblesse (Udekem d'Acoz, 2002 : 15-17).
} 
diplomate. Quant à sa mère, Danièle, elle n'a exercé aucune activité salariée, secondant son mari dans ses tâches. Patrick et Danièle Nothomb sont attachés au respect d'un certain nombre de valeurs et normes propres à l'éducation aristocratique, et qui se caractérise par le cumul d'une série de traits typiques qui ont marqué la socialisation d'Amélie Nothomb, ainsi que celle de son frère et de sa sœur. Deux dimensions prédominent dans cette culture de l'excellence. L'excellence « corporelle » tout d'abord, qui consiste en la retenue des corps et des émotions ${ }^{11}$ et en l'esthétisation du corps, qui doit être mince et droit et dont les femmes doivent plus particulièrement avoir le souci (Pinçon et Pinçon-Charlot, 2000). L'excellence «culturelle » ensuite, qui renvoie à la possession de capital culturel, présent sous la forme de dispositions langagières, d'une culture livresque, de titres scolaires ou encore de biens culturels (Bourdieu, 1979). Mension-Rigau a plus précisément montré que la parole tient une place importante dans la culture lettrée : il interprète « le goût du silence » des aristocrates et des grands bourgeois par le fait que les enquêtés, "habitués à vivre dans un milieu où la seule violence autorisée est celle du langage, ont fortement conscience que les mots peuvent blesser à vie. La sagesse leur dicte d'éviter les mots dits trop promptement et de préférer le silence » (Mension-Rigau, 1997: 209).

Si la parole peut « blesser », c'est parce qu'elle est susceptible d'exercer sur autrui une forme de violence, en particulier dans cette culture de la retenue du corps où le recours à la violence physique est délégitimé. La parole n'a donc pas seulement pour but de divertir mais constitue une arme légitime dont on peut user pour convaincre ou, plus généralement, pour s'imposer dans un rapport de force: Mension-Rigau souligne notamment la valorisation par les aristocrates des « mots d'esprit et formules sèches qui mouchent et font mouche à la fois » (Mension-Rigau, 1997: 220). Patrick Nothomb incarne en pratique cet usage spécifique de la parole à travers ses fonctions de diplomate. En l'observant au cours de dîners mondains organisés à leur domicile, Amélie Nothomb a intériorisé très tôt le fait que les mots ne sont pas neutres et la nécessité de bien maîtriser leur usage :

Je remarquais la diversité du discours de papa en fonction des invités qui venaient à la maison. Ça m'a beaucoup frappée. Et je me suis dit, j'ai des choses à retenir de tout ça, on dit pas la même chose en fonction des gens qui sont là, il ne faut pas choquer les gens, il faut les mettre d'accord euh... faut pas mentir de façon scandaleuse, mais faut mettre beaucoup d'eau dans son vin. (Entretien avec Amélie Nothomb, 17 janvier 2008)

\footnotetext{
${ }^{11}$ Foucault a montré, s'agissant des élites intellectuelles grecques, que la maîtrise de soi est nécessaire à la production et au maintien d'une distinction vis-à-vis de ceux qui ne savent pas se contraindre de leur propre chef (Foucault, 1984).
} 


\subsection{L'expérience de l'anorexie et de sa sortie}

L'entrée dans la puberté d'Amélie Nothomb correspond à une intensification des attentes parentales orientées vers une dimension de cette culture de l'excellence, l'excellence corporelle : « La beauté au sens conventionnel du terme quoi, il faut être beau, élégant, faut être mince, faut avoir une bonne présentation. [...] J'ai bien vu que quand j'avais douze ans ma mère me disait "Tu dois faire attention, tu dois te regarder, tu dois te peser" » (entretien avec Amélie Nothomb, 28 juin 2007). Cette période s'élabore autour de sa difficulté à se conformer à ces injonctions verbales familiales. À cette époque, Amélie Nothomb met en place une ascèse alimentaire de plus en plus radicale, qui va la conduire à l'anorexie. De telles pratiques peuvent être interprétées au prisme du travail de Muriel Darmon comme un moyen de maîtriser les jugements que portent les autres sur son corps (Darmon, 2003).

Lorsqu'en entretien avec nous, Amélie Nothomb évoque sa sortie de l'anorexie, elle présente l'attitude maternelle - et plus largement familiale - comme un retour à un jugement social, parlant «d'insultes » et «d'humiliations » : "Euh tout de suite ça a recommencé : “T’es grosse, t'es grosse”, alors que, franchement, je peux vous assurer, je l'étais pas. Oh je vous dis pas comme j'ai souffert » (entretien avec Amélie Nothomb, 17 janvier 2008). Dans le regard qu'elle porte sur cet épisode de sa vie, Amélie Nothomb établit souvent un lien entre ces injonctions familiales relatives à son apparence corporelle et le rapport douloureux qu'elle entretient à son corps. Ainsi, pour elle, son entrée dans des pratiques anorexiques est associée à des paroles maternelles: « Toujours est-il que à treize ans et demi, très fortement encouragée par ma mère, j’ai dit "Bon c'est fini tout ça, j'arrête de manger" » (entretien avec Amélie Nothomb, 17 janvier 2008). Ainsi, loin d'être seulement ce par quoi se manifestent des opinions ou encore des sentiments, la parole est peu à peu intériorisée comme un moyen efficace d'exercice de la violence. Un signe de cette croyance dans le pouvoir performatif des mots pourrait également s'observer dans le fait, qu'en 2007, Amélie Nothomb décide de débuter une psychanalyse, dont l'un des principes fondateurs s'appuie sur une disposition à croire au pouvoir thérapeutique de la parole $\mathrm{e}^{12}$.

Il faut avoir conscience que ces propos ne sont pas énoncés dans n'importe quel contexte, mais à l'occasion d'entretiens sociologiques auxquels elle se prête à titre d'écrivaine. Autrement dit, il faut prendre en compte les effets propres au champ littéraire dans la production du discours biographique sur soi. Ces discours participent à son positionnement dans le champ littéraire, à la fabrication d'une certaine image de soi qui emprunte à des figures auctoriales déjà connues dans l'histoire littéraire et qui la légitiment en tant qu'écrivaine. La référence à une situation de souffrance et de

\footnotetext{
${ }^{12}$ Chez Freud, le sujet de la psychanalyse est l'inconscient et l'analyse de la parole serait un moyen de le pénétrer (cf. ses textes sur L'interprétation des rêves (1900), Psychopathologie de la vie quotidienne (1904), Le mot d'esprit et sa relation à l'inconscient (1905)). La mise en mots est appréhendée comme ayant des effets curatifs.
} 
solitude s'observe en effet chez de nombreux écrivains, du Moyen-Âge jusqu'aux années 1840, ce type de présentation de soi contribuant à leur fournir des gains symboliques : «Malheureux (persécuté, mélancolique, démuni, etc.) donc légitime (sensible, sincère, génial, original, etc.) » (Brissette, 2005 : 31-32). Néanmoins, Brissette souligne que loin de n'être qu'un "mythe » littéraire décontextualisé, ces discours correspondent à des situations matérielles bien réelles de la vie d'écrivain. De même, en entretien sociologique, si Amélie Nothomb puise dans ce registre discursif parmi une variété d'autres possibles, c'est bien parce qu'il fait particulièrement sens pour elle, au regard des expériences socialisatrices décrites. Ainsi, sa socialisation familiale semble-t-elle l'avoir conduite à intérioriser l'importance qu'une parole bien maîtrisée est susceptible de revêtir dans les rapports sociaux, ainsi que sa force potentiellement destructrice. Faire l'histoire de ce rapport particulier au langage permet de mieux comprendre les formes particulières que prennent ses créations littéraires en les resituant dans ce contexte social précis.

\section{La mise en scène littéraire du pouvoir des mots}

On a vu qu'Amélie Nothomb conférait à la parole un fort pouvoir, que l'on peut associer à une pluralité d'expériences socialisatrices extralittéraires vécues au sein d'une famille d'aristocrates lettrés. Cette vision du monde est actualisée par l'écrivaine sur la scène littéraire, sous la forme d'une compétence qui s'incarne dans une aisance verbale et un art de gérer les relations avec les critiques, journalistes et lecteurs particulièrement rentables sur la scène médiatique (Saunier, 2015). Cette vision du monde se transpose également dans la forme même à travers laquelle elle écrit. Parmi l'espace des possibles littéraires, c'est la logique conversationnelle qui sature en effet la majorité de ses textes, et la manière particulière dont ces échanges sont mis en scène revient à les considérer comme des sortes de duels verbaux ${ }^{13}$.

On peut plus spécifiquement parler d'écriture «théâtralisée » et ce, dès son premier roman, Hygiène de l'assassin, publié en 1992. Le type d'écriture de ses textes les rapproche du «roman dialogué », sorte de dispositif hybride entre le roman et le théâtre, qui s'impose peu à peu à partir des années 1950 (Boblet, 2003). Il déploie, dans ses accointances avec le langage dramatique, une croyance en la puissance d'interpellation de la parole : "Cette parole "en action" a une puissance pragmatique, elle exerce un effet immédiat sur les relations intersubjectives, qu'elle exprime et aussi qu'elle modifie. Les romans dialogués font triompher la fonction conative du langage

\footnotetext{
${ }^{13}$ II ne s'agit pas ici de céder à un automatisme explicatif simpliste consistant à avancer que les auteurs de culture aristocratique useraient prioritairement de la forme dialoguée ou, à l'inverse, que la forme dialoguée serait l'apanage de ce profil sociologique d'écrivains. Notre propos vise bien plutôt à montrer que chez cette écrivaine précisément, au regard de ses diverses expériences socialisatrices, la forme dialoguée est apparue comme celle qui lui permettait le mieux de traduire son rapport particulier au langage.
} 
et sa performativité. Toute prise de parole fait progresser les relations entre les partenaires, les déplace les uns par rapport aux autres» (Boblet, 2003: 66). Les textes d'Amélie Nothomb mobilisent cette fonction pragmatique de la parole en exploitant ces dialogues argumentatifs qui, par leur succession, permettent à l'histoire de se construire. Au travers de répliques courtes, les protagonistes déploient leurs arguments afin de remporter l'adhésion de l'auditoire sur un rythme haché et soutenu, tel un combat d'escrime à la fois vif et rapide, comme le montre cet extrait de dialogue entre Nina, une jeune journaliste, et Prétextat Tach, un vieux romancier détenant le prix Nobel de littérature dans Hygiène de l'assassin :

- [Nina] Répondez à ma question : pourquoi ce roman est-il inachevé ?

- [Tach] Foutre ciel, vous m'emmerdez! Pourquoi ce roman n'aurait-il pas le droit d'être inachevé ?

- Le droit n'a vraiment rien à voir dans cette histoire. Vous écriviez des faits réels avec une fin réelle : alors, pourquoi ne pas avoir achevé ce roman? Après le meurtre de Léopoldine, vous vous arrêtez dans le vide. Était-il si difficile de boucler l'affaire, d'y mettre un terme en bonne et due forme?

- Difficile ! Apprenez, petite dinde, que rien n'est difficile à écrire pour Prétextat Tach.

- Précisément. Cette non-fin en queue de poisson est d'autant plus absurde.

- Qui êtes-vous pour déterminer l'absurdité de mes décisions?

- Je ne détermine rien, je m'interroge. (HA, 123-124)

À l'instar du langage dramatique (Larthomas, 2001), ces dialogues littéraires peuvent donner une illusion de spontanéité, mais ils sont en réalité écrits, simulés et travaillés par Amélie Nothomb pour produire ce que Boblet appelle des «effets d'argumentations » (Boblet, 2003 : 166). Tout se passe comme s'il s'agissait, dans l'écriture de ces dialogues, d'aller au plus près de ce que la joute verbale peut produire comme efficacité performative. Différentes stratégies argumentatives peuvent donc être relevées, tels l'argument ad hominem ("Apprenez, petite dinde, que rien n'est difficile à écrire pour Prétextat Tach »), le recours à la réfutation à travers des arguments logiques, fondés sur des faits, qui contredisent les propos de l'interlocuteur (« alors, pourquoi... »), ou encore l'usage du discours polémique figuré dans un vocabulaire dépréciatif ("petite dinde ») qui ridiculise l'interlocuteur, et une ponctuation expressive, qui marque l'indignation du locuteur.

Les paroles sont appréhendées comme des actes: l'usage de questions et les incitations à répondre imposent une suite à l'échange dans la mesure où « la force juridique de l'illocutoire [...] construit un contrat entre les sujets, révisé à chaque prise de parole, ce qui dynamise la conversation» (Boblet, 2003: 172). Au terme de leur longue joute orale, telle une enquêtrice, Nina réussit à briser au fur et à mesure les défenses de Tach. Une même tension se déploie dans Péplum, récit presqu'entièrement dialogué (le personnage de la romancière se décrit d'ailleurs comme une "dialoguiste » (Pép., 95)), ou encore Cosmétique de l'ennemi, mettant en scène des affrontements verbaux entre respectivement Celsius et A.N. d'une part, et entre Textor Texel et Jérôme 
Angust d'autre part. Telles qu'elles sont mises en scène par Amélie Nothomb, ces interactions verbales présentent la particularité de déclencher des réponses non verbales radicales. Ainsi, au terme de la joute verbale entre Tach et Nina dans Hygiène de l'assassin, Nina parvient à déstabiliser Tach et finit même par l'étrangler: la symbolique de l'étranglement doit ici être relevée comme une manière d'ôter définitivement à son interlocuteur tout usage de la parole et de le tuer à l'endroit même où leur affrontement se déployait. Quant à Cosmétique de l'ennemi, Jérôme Angust, poussé à bout par les provocations verbales de Textor Texel, «fracass[e] à plusieurs reprises sur le mur » $(\mathrm{CE}, 139)$ la tête de ce dernier. On peut faire l'hypothèse qu'à travers de tels dénouements, est mis en valeur le fait que ces stratégies argumentatives constituent des actes de langage dans leur forme extrême: le personnage n'est pas seulement influencé par le discours de l'autre mais bien conduit, au fil de l'interaction verbale, à perpétrer un acte irrémédiable. Le langage est intimement lié à l'action, contribuant à la faire évoluer.

À l'inverse, ceux qui sont privés de langage se trouvent dans l'incapacité de maîtriser ce qui les entoure (les êtres et les choses). C'est par exemple le cas d'Amélie-san dans Stupeur et tremblements. Dès son arrivée, sa confrontation à l'imposante entreprise Yumimoto la conduit à parler de moins en moins : elle se contente d'hocher la tête. Un peu plus tard, après qu'elle a commis une série de gaffes, son supérieur lui ordonne explicitement d'arrêter de parler japonais. Enfin, elle se retrouve dame-pipi, emploi au cours duquel elle parle de moins en moins. Dans une telle situation, elle en vient peu à peu à perdre la maîtrise du temps et s'identifie à son travail, sans être en mesure de le mettre à distance :

Les mois passèrent. Chaque jour, le temps perdait de sa consistance. J'étais incapable de déterminer s'il s'écoulait vite ou lentement. Ma mémoire commençait à fonctionner comme une chasse d'eau. Je la tirais le soir. Une brosse mentale éliminait les dernières traces de souillure. (ST, 161-162)

La perte du langage se fait donc à la fois marque de la domination et canal par lequel la domination s'exerce. Les personnages dépourvus de la maîtrise du langage ont peu de pouvoir sur autrui. Cette vision qu'a l'écrivaine de la performativité de la parole s'actualiserait ainsi dans sa pratique d'écriture en se manifestant dans cette forme d'écriture " théâtralisée » ${ }^{14}$.

Reste que ces formes langagières ne sont pas toutes dotées du même statut symbolique et de la même efficacité. Directement relié au cas de joute verbale précédent, c'est le langage verbal argumentatif et économe en mots qui se trouve le plus souvent valorisé, la figure repoussoir étant le bavardage, correspondant en cela aux représentations de l'excellence langagière qu'Amélie Nothomb a intériorisées au cours de sa socialisation familiale. Dans Mercure, la conversation peut "émanciper » et « libérer » (Mer., 40), tandis que le « bavardage » est perçu comme une « logorrhée » qui

${ }^{14}$ On comprend dès lors mieux pourquoi les textes d'Amélie Nothomb ont été autant adaptés au théâtre, car leur forme s'y prête particulièrement bien. 
« envahit » et emprisonne (Mer., 40). En outre, le silence est considéré comme supérieur aux mots. Il faut donc savoir se taire. Dans ce texte, Hazel et Françoise valorisent les personnes qui savent rester silencieuses, le silence les rendant « intéressant[es] (Mer., 39), car «mystérieu[ses]» (Mer., 39). Matthew est initialement présenté par Hazel comme un personnage «intéressant » (Mer., 39) du fait de son silence : en lisant son devoir devant la classe (« un récit assez bavard où il racontait ses vacances aux sports d'hiver» (Mer, 39)), il perd alors tout intérêt à ses yeux. Zdena établit la même distinction dans Acide sulfurique. D'un côté, figurent les discours de Pannonique et de son compagnon EPJ 327 qui produisent un effet sur l'interlocuteur : « Une "chose à dire" était une parole où rien n'était superflu et où il était échangé des informations si essentielles que l'autre en était marqué pour toujours» (AS, 112). Les paroles de Pannonique sont ainsi considérées comme des paroles « chocs » (AS, 112). À l'autre pôle se trouve le bavardage, un « blabla », un « ronronnement » (AS, 111) qui ressemble à un échange sans en être vraiment un (« la vacuité de leurs monologues alternés » (AS, 111)). De même, dans Hygiène de l'assassin, le silence de Tach «embarrasse » (HA, 10) les journalistes venus l'interviewer. Tach est défini par eux comme une personne prestigieuse et un « génie » (HA, 25). La retenue de ses émotions corporelles et le son de sa voix incarnent la domination. Un des journalistes souligne son «visage lisse et glacial de mépris » (HA, 24) et le compare à un « empereur » (HA, 24). Non sans humour, Amélie Nothomb présente ces journalistes comme admirant la " concision dans la méchanceté » (HA, 25) dont Tach fait preuve en entretien avec eux. Marque des dominants dans ce contexte, le silence s'inscrit du côté de ceux qui n'ont pas besoin de parler pour prouver et défendre leur valeur: par exemple, dans Acide sulfurique, malgré la pression de ses camarades, Pannonique refuse de justifier le fait qu'elle ne souhaite pas céder à Zdena pour sauver ses camarades.

\section{Conclusion}

La mise en série des textes conduit à instituer des différences dans les divers types de langage traités. La distinction semble plus précisément se réaliser selon une vision élitiste du langage, que l'on pourrait situer dans le prolongement des expériences socialisatrices d'Amélie Nothomb, où la maîtrise du langage et sa retenue sont censées marquer la supériorité sociale et/ou manifester la hauteur d'esprit. Cette étude d'une dimension des textes d'Amélie Nothomb, bien que rapide, permet néanmoins de montrer que des éléments extérieurs à l'univers littéraire, hérités de socialisations antérieures, viennent «agir» sur des pratiques littéraires. Le rapport spécifique qu'Amélie Nothomb entretient avec les mots se traduit dans le choix d'une écriture « théâtralisée » ainsi que dans les types de situations mises en scène que nous avons décrites. Des raisonnements similaires ont déjà pu être observés dans des recherches antérieures relatives à d'autres domaines professionnels : on pense notamment aux travaux de Baszanger qui montrent, malgré la dimension fortement balisée de la 
formation et de la profession médicales, la «diversité de [cette] profession », et le fait que la trajectoire des professionnels « est constamment pénétrée par des éléments qui ont leur origine ailleurs [...] et qui viennent informer différemment ce territoire commun » (Baszanger, 1981: 228-229). L'intérêt de travailler sur la sphère littéraire permet de rendre compte des effets des socialisations antérieures ou parallèles jusque dans la pratique même d'écriture, appréhendable à travers son contenu stylistique et thématique.

Ne pourrait-on alors pas généraliser le type de raisonnement mobilisé sur cette activité professionnelle-artistique à d'autres domaines de la pratique de la vie sociale, en établissant une sorte de théorie de la transposition et de ses codes ? Comme le souligne Lahire, le rapport entre l'expérience et le produit de la pratique n'est pas de même « nature » en fonction des domaines, et certains univers (tel le jeu littéraire) offrent davantage la possibilité que d'autres de transposer certaines expériences personnelles (Lahire, 2010: 87-91). Il n'en reste pas moins qu'une telle entreprise mériterait d'être explorée afin d'entrer, avec peut-être encore plus d'acuité, dans la compréhension des « actes » des individus au sens large (que ce soit des discours, la production d'écrits ou encore des pratiques). Cela nécessiterait de réfléchir au type de transposition spécifique à l'univers en question (et donc de suffisamment bien le connaître pour être en mesure de repérer, derrière des pratiques d'individus qui peuvent parfois sembler identiques ou standardisées, des «styles » différents), et d'étudier finement ce qui se transpose.

\section{Bibliographie}

Austin J. (1991 [1962]), Quand dire, c'est faire, Paris, Seuil, (« Point essais »).

BARTHES R. (1966), «Introduction à l’analyse structurale des récits », Communications, $\mathrm{n}^{\circ} 8$, p. 1-27.

BASZANGER I. (1981), "Socialisation professionnelle et contrôle social. Le cas des étudiants en médecine futurs généralistes », Revue française de sociologie, vol. 22, $n^{\circ} 2$, p. 223-245.

Berger P., LuCKMANN T. (1996 [1986]), La Construction sociale de la réalité, Paris, Méridiens Klincksieck.

Berthelot J.-M., Foulon A., Ponthieux S. (1992), Les enseignants-chercheurs de l'enseignement supérieur: revenus professionnels et conditions d'activité, Paris, La Documentation française/CERC.

Boblet M.-H. (2003), Le roman dialogué après 1950. Poétique de l'hybridité, Paris, Honoré Champion. 
Boschetti A. (2001), La poésie partout. Apollinaire, homme-époque (1898-1918), Paris, Seuil (« Liber »).

BOURDIEU P. (1979), «Les trois états du capital culturel », Actes de la recherche en sciences sociales, $\mathrm{n}^{\circ} 30$, p. 3-6.

BOURDIEU P. (1992), Les règles de l'art, genèse et structure du champ littéraire, Paris, Seuil.

BRISSETTE P. (2005), La malédiction littéraire : du poète crotté au génie malheureux, Montréal, Presses de l’Université de Montréal (« Socius »).

DARMOn M. (2003), Devenir anorexique, une approche sociologique, Paris, La Découverte.

DARMon M. (2006), La socialisation, Paris, Armand Colin («128»).

Elias N. (1991), Mozart. Sociologie d'un génie, trad. fr., Paris, Seuil.

FABIANI J.-L. (1999), « Les règles du champ », in B. LAHIRE (dir.), Le Travail sociologique de Pierre Bourdieu. Dettes et critiques, Paris, La Découverte, p. 75-91.

Foucault M. (1984), Histoire de la sexualité. II. L’usage des plaisirs, Paris, Gallimard.

GIRAUD F., SAUNIER E. (2012), « La posture littéraire à l'épreuve de deux cas empiriques. Pour une prise en compte des expériences extralittéraires des écrivains ", COnTEXTES [en ligne], Varia, mis en ligne le 24 janvier 2012. URL: http://journals.openedition.org/contextes/4892.

LAHIRE B. (2006), La condition littéraire, la double vie des écrivains, Paris, La Découverte.

LAHIRE B. (2010), Franz Kafka, éléments pour une théorie de la création littéraire, Paris, La Découverte.

Larthomas P. (2001), Le langage dramatique. Sa nature, ses procédés, Paris, PUF (« Quadrige »).

MEIzOZ J. (2007), Postures littéraires. Mises en scènes modernes de l'auteur, Genève, Stalkine (" Érudition »).

MENGer P.-M. (2009), Le travail créateur, s'accomplir dans l'incertain, Paris, SeuilGallimard (« Hautes Études»).

MENSION-Rigau É. (1997), Aristocrates et grands bourgeois, éducation, traditions, valeurs, Paris, Perrin.

PANOFSKy E. (1967), Architecture gothique et pensée scolastique, Paris, Minuit (« Le Sens commun »).

Pinçon M., Pinçon-Charlot M. (2000), Sociologie de la bourgeoisie, Paris, La Découverte (« Repères »).

Propp V. (1965), Morphologie du conte, Paris, Seuil. 
SAUNIER É. (2012), L'écriture comme objectivation et travail de soi dans les cuvres d'Amélie Nothomb : pour une approche sociologique de la création littéraire, thèse de doctorat en sociologie, Lyon, Université Lumière Lyon 2.

SAUNIER É. (2015a), « Accéder à la reconnaissance en tant que femme écrivain belge : une étude du cas d'Amélie Nothomb dans le champ littéraire français », Sociologie et sociétés, vol. $47, \mathrm{n}^{\circ} 2$, p. 101-123.

SAUNIER É. (2015b), « Produire la valeur artistique dans une économie de la notoriété. Le cas d'Amélie Nothomb, Terrains et travaux, vol. 26, n 1, p. 41-61.

Udekem D’Acoz (d') M.-P. (2002), Pour le Roi et la Patrie. La noblesse belge dans la Résistance, Bruxelles, Éditions Racine.

VIART D. (2007), «Littérature et sociologie, les champs du dialogue », in P. BAUDORRE, D. RABATE, D. VIART (dir.), Littérature et sociologie, Bordeaux, Presses Universitaires de Bordeaux ("Sémaphores »).

WEBER M. (1995), Économie et société. I. Les catégories de la sociologie, Paris, Plon (« Agora Pocket »).

\section{Corpus des textes étudiés}

(1992), Hygiène de l'assassin, Paris, Albin Michel.

(1993), Le Sabotage amoureux, Paris, Albin Michel.

(1994), Les Combustibles, Paris, Albin Michel.

(1995), Les Catilinaires, Paris, Albin Michel.

(1996), Péplum, Paris, Albin Michel.

(1997), Attentat, Paris, Albin Michel.

(1998), Mercure, Paris, Albin Michel.

(1999), Stupeur et tremblements, Paris, Albin Michel.

(2000), Métaphysique des tubes, Paris, Albin Michel.

(2001), Cosmétique de l'ennemi, Paris, Albin Michel.

(2002), Robert des noms propres, Paris, Albin Michel.

(2003), Antéchrista, Paris, Albin Michel.

(2004), Biographie de la faim, Paris, Albin Michel.

(2005), Acide sulfurique, Paris, Albin Michel. 
(2006), Journal d'Hirondelle, Paris, Albin Michel.

(2007), Ni d’Ève, ni d’Adam, Paris, Albin Michel.

\section{Liste des abréviations des romans d'Amélie Nothomb cités dans cet article}

AS : Acide sulfurique. Pagination de l'édition Albin Michel.

CE : Cosmétique de l'ennemi. Pagination de l'édition Albin Michel.

HA : Hygiène de l'assassin. Pagination en collection « Points».

Mer. : Mercure. Pagination du Livre de Poche.

Pép. : Péplum. Pagination du Livre de Poche. 